\title{
Erratum to: Physiological and metabolic analysis of nitrate reduction on poly-gamma-glutamic acid synthesis in Bacillus licheniformis WX-02
}

\author{
Xin Li $\cdot$ Xiangyong Gou $\cdot$ Dan Long $\cdot$ Zhixia Ji $\cdot$ \\ Lifang Hu $\cdot$ Dihong Xu $\cdot$ Jun Liu $\cdot$ Shouwen Chen
}

Published online: 1 October 2014

(C) Springer-Verlag Berlin Heidelberg 2014

\section{Erratum to: Arch Microbiol}

DOI 10.1007/s00203-014-1014-y

In the original publication, the corresponding author was wrongly published as Dr. Xin Li. The correct corresponding author for this article is "Pro. Shouwen Chen".

The online version of the original article can be found under doi:10.1007/s00203-014-1014-y.

X. Li $\cdot$ X. Gou $\cdot$ D. Long $\cdot$ Z. Ji $\cdot$ L. Hu $\cdot$ S. Chen $(\bowtie)$ State Key Laboratory of Agricultural Microbiology, College of Life Science and Technology, Huazhong Agricultural University, Wuhan 430070, People's Republic of China e-mail: chenshouwen@mail.hzau.edu.cn

X. Li

e-mail: lixin0807@gmail.com

X. Li

Institute of Biological Engineering, Hubei University of Technology, Wuhan 430068, People's Republic of China

D. Xu

Institute of Technology, Huazhong Agricultural University,

Wuhan 430070, People's Republic of China

\section{J. Liu}

School of Biology and Pharmaceutical Engineering,

Wuhan Polytechnic University, Wuhan 430023,

People's Republic of China

S. Chen

Hubei Collaborative Innovation Center for Green Transformation of Bio-Resources, College of Life Sciences, Hubei University,

Wuhan 430062, People's Republic of China 